\title{
Post-placement Rewiring by Exhaustive Search for Functional Symmetries
}

\author{
Kai-hui Chang, Igor L. Markov and Valeria Bertacco \\ University of Michigan, Ann Arbor
}

\begin{abstract}
We propose two new algorithms for rewiring - a post-placement optimization that reconnects pins of a given netlist without changing the logic function and gate locations. In the first algorithm, we extract small sub-circuits consisting of several gates from the design and reconnect pins according to the symmetries of the sub-circuits. To enhance the power of symmetry detection, we also propose a graph-based symmetry detector that can identify permutational and phase-shift symmetries on multiple input and output wires, as well as hybrid symmetries, creating abundant opportunities for rewiring. Our second algorithm, called long-range rewiring, is based on reconnecting equivalent pins and can augment the first approach for further optimization. We apply our techniques for wirelength optimization and observe that it provides wirelength reduction comparable to that achieved by detailed placement.
\end{abstract}

Categories and Subject Descriptors: B.7.2 [Hardware]: Integrated Circuits—Design Aids

General Terms: Algorithms, Design, Performance

Additional Key Words and Phrases: VLSI, Placement, Rewiring

\section{INTRODUCTION}

The semiconductor industry's trend of always pushing the limit for minimum transistor sizing has led to new challenges in integrated circuit design. One key challenge is wire parasitics, whose aggravation is leading to increased delay and power consumption in wires, to the point that their contribution dominates over the ones from transistor switching. Since accurate wire topology is available only after the circuit is placed, post-placement optimizations have been studied extensively. Typically, these optimizations focus on improving delay, power, reliability and/or wirelength. However, we observe that existing techniques may fail to provide consistent improvements because, when optimizing one design aspect, they may worsen other physical aspects. For example, cell cloning may improve timing at the expense of significantly increasing wirelength [Hrkic et al. 2004], and the timing optimization may actually worsen the final delay because of critical path detour after routing. The widespread unpredictability of all these optimizations makes design closure an extremely difficult task, often leading to many iterations of post-placement optimization and to delays in the design completion.

In general, the stability, or lack thereof, of an optimization can be determined by checking if it creates illegal changes to the layout. For instance, if an optimization changes placement by creating cell overlaps, then an additional legalization step needs to be performed to remove the overlaps, and the latter could cancel out or even worsen the optimizations of the former. As a specific example, rewiring techniques based on addition and removal of wires may create overlaps because cell types will be changed [Chang and Marek-Sadowska 2001; Chang et al. 1997; Chang et al. 1999; Jiang et al. 1997; Wu et al. 2000], and the evaluation of the changes must be delayed until legalization is performed. On the other hand, the impact of optimizations that do not affect cell locations can be evaluated immediately and reliably, therefore they have little risk of hampering the design closure. In addition, such optimizations allow metal fix, a post-silicon repair technique that only changes the metal layers while preserving the masks for manufacturing transistors. With the mask cost approaching one million dollars per set, being able to repair a circuit by metal fix reduces the cost of respin significantly.

K.-H. Chang et al. [2006] pointed out that symmetry-based rewiring is the only post-placement optimization that does not affect cell placement. As a result, it can be integrated into any design flow safely, and it allows metal fix. To this end, the symmetry-based rewiring system proposed by C.-W. Chang et al. [2004] is effective in optimizing

Author's address: K.-H. Chang, I.L. Markov and V. Bertacco, University of Michigan, EECS Department, 2260 Hayward Ave., Ann Arbor, MI 48109-2121. Email \{changkh, imarkov, valeria\}@eecs. umich. edu

Permission to make digital/hard copy of all or part of this material without fee for personal or classroom use provided that the copies are not made or distributed for profit or commercial advantage, the ACM copyright/server notice, the title of the publication, and its date appear, and notice is given that copying is by permission of the ACM, Inc. To copy otherwise, to republish, to post on servers, or to redistribute to lists requires prior specific permission and/or a fee.

(c) 2007 ACM 1529-3785/2007/0700-0001 $\$ 5.00$ 
delay, power and reliability of a digital circuit. Their system iteratively extracts subcircuits from the circuit and performs rewiring optimizations by exploiting symmetries. However, their symmetry-detection technique is only applicable to designs synthesized using certain basic gate types and cannot be applied to many practical circuits.

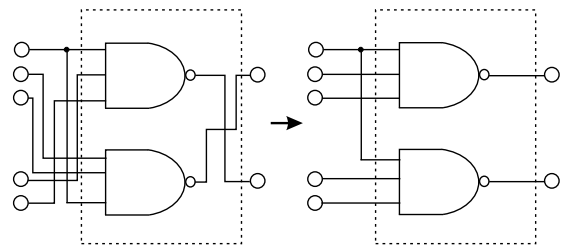

(a)

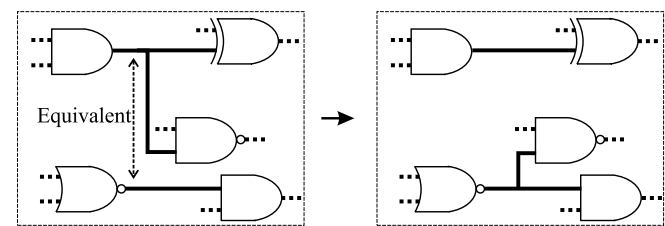

(b)

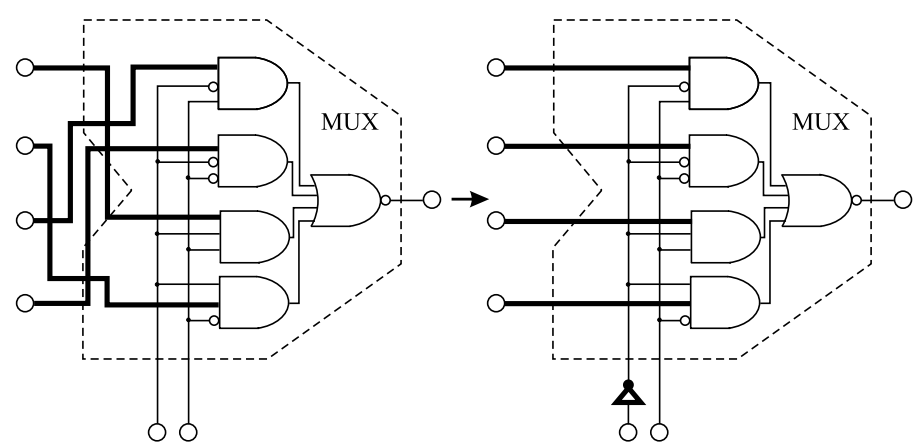

(c)

Fig. 1. Rewiring examples: (a) multiple inputs and outputs are rewired simultaneously using pin-permutation symmetry, (b) long-range rewiring using equivalent wires, (c) two inputs to a multiplexer are rewired by inverting one of the select bits. Bold lines represent changes made in the circuit.

In this work we extend our preliminary work in [Chang et al. 2005] and propose a novel rewiring technique that can be applied to designs mapped to any type of cells. This is achieved by detecting symmetries in Boolean functions regardless of the logic structure used to represent them. In addition, we observe that detecting fewer symmetries creates fewer possibilities for optimization. While previous work on rewiring considers input symmetries only, our comprehensive symmetry detector allows us to handle both input and output permutations, as well as their composition. For example, the rewiring opportunity in Figure 1(a) cannot be discovered unless both input and output symmetries can be detected. In addition, "phase-shift" symmetries [Kravets and Sakallah 2000], that is, symmetries involving negation of inputs and/or outputs, can also be detected. An example of this application is given in Figure 1(c). In our rewiring approach, we extract subcircuits iteratively from the circuit, and we use the symmetries detected to reconnect pins and optimize wirelength. We also propose a "long-range" rewiring technique which operates by rearranging the connection of equivalent pins, complementing the baseline method (an example is shown in Figure 1(b)). Long-range rewiring first identifies candidate equivalent wires by random simulation and uses formal methods to verify their equivalency. Similar to symmetry-based rewiring, it does not affect cell placement. Our experimental results show that the proposed approach can reduce total wirelength by approximately $5 \%$. While this new symmetry-detection technique is potentially less scalable than previous ones, we found experimentally that the execution runtimes were reasonable.

The remainder of the paper is organized as follows. Section 2 introduces basic principles of symmetry, and describes relevant previous work on symmetry detection and circuit rewiring. In Section 3 we describe our symmetrydetection algorithm. Section 4 discusses the post-placement rewiring algorithms. Finally, we provide experimental results in Section 5 and conclude in Section 6.

\section{BACKGROUND}

The rewiring technique proposed in this paper is based on symmetry detection. Therefore, in this section, we present background ideas and related work about symmetry detection. Previous work on post-placement rewiring is also discussed.

\subsection{Symmetries in Boolean Functions}

One can distinguish semantic (functional) symmetries of Boolean functions from the symmetries of specific representations (syntactic symmetries). All syntactic symmetries are also semantic, but not vice versa. For example, in function " $o=(x+y)+z$ ", $x \leftrightarrow z$ is a semantic symmetry because the function will not be changed after the 
permutation of variables; however, it is not a syntactic symmetry because the structure of the function will be changed. On the other hand, $x \leftrightarrow y$ is both a semantic and syntactic symmetry. In this work we exploit functional symmetries, whose definition is provided below.

DEFINITION 1. Consider a multi-output Boolean function $F: \mathcal{B}^{n} \rightarrow \mathcal{B}^{m}$, where

$$
F\left(i_{1} \ldots i_{n}\right)=<f_{1}\left(i_{1} \ldots i_{n}\right), f_{2}\left(i_{1} \ldots i_{n}\right) \ldots f_{m}\left(i_{1} \ldots i_{n}\right)>.
$$

A functional symmetry is a one-to-one mapping $s: \mathcal{B}^{(n+m)} \rightarrow \mathcal{B}^{(n+m)}$ such that:

$$
<f_{1}\left(i_{1} \ldots i_{n}\right), f_{2}\left(i_{1} \ldots i_{n}\right) \ldots f_{m}\left(i_{1} \ldots i_{n}\right)>=<s\left(f_{1}\right)\left(s\left(i_{1}\right) \ldots s\left(i_{n}\right)\right), s\left(f_{2}\right)\left(s\left(i_{1}\right) \ldots s\left(i_{n}\right)\right) \ldots s\left(f_{m}\right)\left(s\left(i_{1}\right) \ldots s\left(i_{n}\right)\right)>.
$$

In other words, a functional (semantic) symmetry is a transformation of inputs and outputs which does not change the functional relation between them.

EXAMPLE 1. Consider the multi-output function $z=x_{1}$ XOR $y_{1}$ and $w=x_{2}$ XOR $y_{2}$. The variable-permutation symmetries include: (1) $x_{1} \leftrightarrow y_{1}$, (2) $x_{2} \leftrightarrow y_{2}$, (3) $x_{1} \leftrightarrow x_{2}, y_{1} \leftrightarrow y_{2}$, and $z \leftrightarrow w$ (all swaps are performed simultaneously). In fact, all the symmetries of this function can be generated from combinations of the symmetries listed above. A set of symmetries with this property are called "symmetry generators". For example, the symmetry “ $x_{1} \leftrightarrow y_{2}, y_{1} \leftrightarrow x_{2}$, and $z \leftrightarrow w$ " can be generated by applying the symmetries (1), (2) and (3).

While most previous work on symmetry detection focuses on permutations of two variables, Pomeranz and Reddy [1994] and Kravets and Sakallah [2000] consider swaps of groups of ordered variables. These swaps are called higher-order symmetries in [Kravets and Sakallah 2000]. For example, if variables $a, b, c$ and $d$ in the support of function $f$ satisfy the condition:

$$
F(. ., a, . ., b, . ., c, . ., d, . .)=F(. ., c, . ., d, . ., a, . ., b, . .)
$$

then we say that $f$ has a second-order symmetry between ordered variable groups $(a, b)$ and $(c, d)$. Such higherorder symmetries are common in realistic designs. For example, in a 4-bit adder, all bits of the two input numbers can be swapped as groups (preserving the order of the bits), but no two input bits in different bit positions are symmetric by themselves. Kravets also introduced phase-shift symmetry as a function-preserving transformation involving the inversion of one or more inputs that do not permute any of the inputs. Our work generalizes this concept by including output symmetries involving inversion in the class of phase-shift symmetries. We also define composite phase-shift symmetry as a symmetry which consists of phase-shift and permutational symmetries.

In this paper we commonly refer to composite phase-shift symmetries as just phase-shift symmetries, except for pure phase-shift symmetries which do not include permutations.

EXAMPLE 2. Consider again the multi-output function $z=x_{1}$ XOR $y_{1}$ and $w=x_{2}$ XOR $y_{2}$ given in Example 1. Aside from the pin-swap symmetries discussed in Example 1, the following phase-shift symmetries also exist in the circuit: (1) $x_{2} \leftrightarrow y_{2}^{\prime}$, (2) $x_{1} \leftrightarrow y_{1}^{\prime}$, (3) $x_{2} \leftrightarrow x_{2}^{\prime}$ and $w \leftrightarrow w^{\prime}$, (4) $x_{1} \leftrightarrow x_{1}^{\prime}$ and $z \leftrightarrow z^{\prime}$. Among these symmetries, (1) and (2) are composite phase-shift symmetries because they involve both inversion and permutation of inputs, while (3) and (4) are pure phase-shift symmetries because only inversions of inputs and outputs are used. Note that due to Boolean consistency, a symmetry composed of complement of variables in another symmetry is the same symmetry. For example, $y_{2} \leftrightarrow x_{2}^{\prime}$ is the same as $x_{2} \leftrightarrow y_{2}^{\prime}$.

\subsection{Semantic and Syntactic Symmetry Detection}

Symmetry detection in Boolean functions has several applications, including technology mapping, technologyindependent logic synthesis, BDD minimization [Panda et al. 1994] and circuit rewiring [Chang et al. 2004]. Methods for symmetry detection can be classified into four categories: BDD-based, graph-based, circuit-based and Boolean matching based. However, it is relatively difficult to find all symmetries of a Boolean function regardless of the representation used.

BDDs are particularly convenient for semantic symmetry detection because they support abstract functional operations. One naive way to find two-variable symmetries is to compute the cofactors for every pair of variables, say they are $v_{1}$ and $v_{2}$, and check if $F_{\overline{v_{1}} v_{2}}=F_{v_{1} \overline{v_{2}}}$ or $F_{\overline{v_{1}}} \overline{v_{2}}=F_{v_{1} v_{2}}$. Recent research [Mishchenko 2003] indicates that symmetries can be found or disproved without computing all the cofactors and thus significantly speeds up symmetry detection. However, work on BDD-based symmetry detection has been limited to input permutations only, probably due to the single-output nature of BDDs.

In this paper, symmetry-detection methods that rely on efficient algorithms for the graph automorphism problem (i.e., finding all symmetries of a given graph) are classified as graph-based. They construct a graph whose symmetries faithfully capture the symmetries of the original object, find its automorphisms (symmetries) and map them back to the original object. Aloul et al. [2003] proposed a way to find symmetries for SAT clauses using this approach. The symmetry-detection approach proposed in this paper is inspired by their work. 
Circuit-based symmetry-detection methods often convert a circuit representing the function in question to a more regular form, where symmetry detection is more practical and efficient. For example, Wang et al. [2003] transform the circuit to NOR gates. C.-W. Chang et al. [2004] use a more elaborate approach by converting the circuit to XOR, AND, OR, INV and BUF first, and then partition the circuit so that each subcircuit is fanout free. Next, they form "supergates" from the gates and detect symmetries for those supergates. Wllace [2001] uses concepts from Boolean decomposition [Bertacco and Damiani 1997] and converts the circuit to "quasi-canonical forms", and then input symmetries are recognized from these forms. This technique is capable of finding higherorder symmetries. Another type of circuit-based symmetry detector relies on ATPG and simulation, such as the work by Pomeranz and Reddy [1994]. Although their technique was developed to find both first and higher order symmetries, they reported experimental results for first-order symmetries only. Therefore its capability to detect higher order symmetries is unclear.

Boolean matching is a problem related to symmetry detection. Its purpose is to compute a canonical representation for Boolean functions that are equivalent under negation and permutation of inputs and outputs. Symmetries are implicitly processed by Boolean matching in that all functions symmetric to each other will have the same canonical representation. However, enumerating symmetries from Boolean matching is not straight forward and requires extra work. This topic has been studied by Wu et al. [1994] and Chai and Kuehlmann [2005].

Table I. A comparison of different symmetry-detection methods. In the table, $n$ is the number of inputs to the circuit and $m$ is the number of gates. Currently known BDD-based and most circuit-based methods can detect only a fraction of all symmetries in some cases, while the method based on graph automorphism (this work) can detect all symmetries exhaustively. Additionally, the symmetry-detection techniques in this work find all phase-shift symmetries as well as composite (hybrid) symmetries that simultaneously involve both permutations and phase-shifts. In contrast, existing literature on functional symmetries does not consider such composite symmetries.

\begin{tabular}{|c|c|c|c|c|}
\hline Data structure used & Target & Symmetries detected & Main applications & $\begin{array}{l}\text { Time com- } \\
\text { plexity }\end{array}$ \\
\hline BDD [Mishchenko 2003] & Boolean functions & All $1^{\text {st }}$ order input symmetries & Synthesis & $\mathrm{O}\left(n^{3}\right)$ \\
\hline $\begin{array}{l}\text { Circuit - Supergate } \\
\text { [Chang et al. 2004] }\end{array}$ & Gate-level circuits & $\begin{array}{l}1^{\text {st }} \text { order input symmetries in supergates, } \\
\text { opportunistically }\end{array}$ & $\begin{array}{l}\text { Rewiring, technology } \\
\text { mapping }\end{array}$ & $\mathrm{O}(m)$ \\
\hline $\begin{array}{l}\text { Circuit - Boolean decom- } \\
\text { position [Wllace 2001] }\end{array}$ & Gate-level circuits & $\begin{array}{l}\text { Input and output permutational symme- } \\
\text { tries, higher-order }\end{array}$ & $\begin{array}{l}\text { Rewiring, physical de- } \\
\text { sign }\end{array}$ & $\Omega(m)$ \\
\hline $\begin{array}{l}\text { Circuit - simulation, } \\
\text { ATPG [Pomeranz and } \\
\text { Reddy 1994] }\end{array}$ & Gate-level circuits & $\begin{array}{l}\text { Input, output and phase-shift symmetries, } \\
\text { higher-order }\end{array}$ & $\begin{array}{l}\text { Error diagnosis, technol- } \\
\text { ogy mapping }\end{array}$ & $\Omega\left(2^{n}\right)$ \\
\hline $\begin{array}{l}\text { Boolean matching [Chai } \\
\text { and Kuehlmann 2005] }\end{array}$ & Boolean functions & $\begin{array}{l}\text { Input, output and phase-shift symmetries, } \\
\text { higher-order }\end{array}$ & Technology mapping & $\Omega\left(2^{n}\right)$ \\
\hline $\begin{array}{l}\text { Graph automorphism } \\
\text { (this work) }\end{array}$ & $\begin{array}{l}\text { Both (with small } \\
\text { number of inputs) }\end{array}$ & $\begin{array}{l}\text { All input, output, phase-shift symmetries } \\
\text { and all orders, exhaustively }\end{array}$ & $\begin{array}{l}\text { Exhaustive small group } \\
\text { rewiring }\end{array}$ & $\Omega\left(2^{n}\right)$ \\
\hline
\end{tabular}

A comparison of BDD-based symmetry detection [Mishchenko 2003], circuit-based symmetry detection [Chang et al. 2004; Pomeranz and Reddy 1994; Wllace 2001], Boolean matching based symmetry detection [Chai and Kuehlmann 2005] and the method proposed in this paper is summarized in Table I.

\subsection{Graph Automorphism Algorithms}

Our symmetry-detection method is based on efficient graph automorphism algorithms, which have recently been improved by Darga et al. [2004]. Their symmetry detector Saucy finds all symmetries of a given colored undirected graph. To this end, consider an undirected graph $G$ with $n$ vertices, and let $V=\{0, \ldots, n-1\}$. Each vertex in $G$ is labeled with a unique value in $V$. A permutation on $V$ is a bijection $\pi: V \rightarrow V$. An automorphism of $G$ is a permutation $\pi$ of the labels assigned to vertices in $G$ such that $\pi(G)=G$; we say that $\pi$ is a structure-preserving mapping or symmetry. The set of all such valid relabellings is called the automorphism group of $G$. A coloring is a restriction on the permutation of vertices - only vertices in the same color can map to each other. Given $G$, possibly with colored vertices, Saucy produces symmetry generators that form a compact description of all symmetries. Saucy is available online at [Saucy ].

\subsection{Post-placement Rewiring}

Rewiring based on symmetries can be used to optimize circuit characteristics. Some rewiring examples are illustrated in Figure 1(a) and 1(c). Here the goal is to reduce wirelength, and swapping symmetric input and output pins accomplishes this.

C.-W. Chang et al. [2004] use the symmetry-detection technique described above to optimize delay, power, and reliability. In general, the symmetry detection in their work is done opportunistically rather than exhaustively. 
Experimental results show that their approach can achieve these goals effectively using the symmetries detected. However, they cannot find the rewiring opportunity in Figure 1(a) and 1(c) because their symmetry-detection technique lacks the ability to detect output and phase-shift symmetries.

Another type of rewiring is based on the addition and removal of wires. Three major techniques are used to determine the wires that can be reconnected. The first one uses reasoning based on ATPG (Automatic Test Pattern Generation) such as REWIRE [Chang et al. 1999], RAMFIRE [Chang and Marek-Sadowska 2001] and the work by Jiang et al. [1997], which tries to add a redundant wire that makes the target wire redundant so that it can be removed. The second class of techniques is graph-based; one example is GBAW [Wu et al. 2000], which uses pre-defined graph representation of subcircuits and relies on pattern matching to replace wires. The third technique uses SPFDs (Sets of Pairs of functions to be Distinguished) [Cong and Long 2001] and is based on don't-cares. Although these techniques are potentially more powerful than symmetry-based rewiring because they allow more aggressive layout change, they are also less stable and do not support post-silicon metal fix.

\section{EXHAUSTIVE SEARCH FOR FUNCTIONAL SYMMETRIES}

The symmetry-detection method presented in our work can find all input, output, multi-variable and phase-shift symmetries including composite (hybrid) symmetries. It relies on symmetry detection of graphs, thus the original Boolean function must be converted to a graph first. After that, it solves the graph automorphism (symmetry detection) problem on this graph, and then the symmetries found are converted to symmetries of the original Boolean function. Our main contribution is the mapping from a Boolean function to a graph, and showing how to use it to find symmetries of the Boolean function. These techniques are described in detail in this section.

\subsection{Problem Mapping}

To reduce functional symmetry detection to the graph automorphism problem, we represent Boolean functions by graphs as described below:

(1) Each input and its complement are represented by two vertices in the graph, and there is an edge between them to maintain Boolean consistency (i.e. $x \leftrightarrow y$ and $x^{\prime} \leftrightarrow y^{\prime}$ must happen simultaneously). These vertices are called input vertices. Outputs are handled similarly, and the vertices are called output vertices.

(2) Each minterm and maxterm of the Boolean function is represented by a term vertex. We introduce an edge connecting every minterm vertex to the output and an edge connecting every maxterm vertex to the complement of the output. We also introduce an edge between every term vertex and every input vertex or its complement, depending on whether that input is 1 or 0 in the term.

(3) Since inputs and outputs are bipartite-permutable, all input vertices have one color, and all outputs vertices have another color. All term vertices use yet another color.

The idea behind this construction is that if an input vertex gets permuted with another input vertex, the term vertices connected to them will also need to be permuted. However, the edges between term vertices and output vertices restrict such permutations to the following cases: (1) the permutation of term vertices does not affect the connections to output vertices, which means the outputs are unchanged, (2) permuting term vertices may also require permuting output vertices, thus capturing output symmetries. A proof of correctness appears in the Appendix.

Figure 2(a) shows the truth table of function $z=x \oplus y$, and Figure 2(b) illustrates our construction for the function. In general, vertex indices are assigned as follows. For $n$ inputs and $m$ outputs, the $i$ th input is represented by vertex $2 i$, while the complementary vertex has index $2 i+1$. There are $2^{n}$ terms, and the $i$ th term is indexed by $2 n+i$. Similarly, the $i$ th output is indexed by $2 n+2^{n}+2 i$, while its complement is indexed by $2 n+2^{n}+2 i+1$.

The symmetry detector Saucy [Darga et al. 2004] used in this work typically runs faster when the graph is smaller and contains more colors. Therefore if output symmetries do not need to be detected, a reduced version of the graph can be used. It is constructed similarly to the full graph, but without output vertices and potentially with more vertex colors. We define an output pattern as a set of output vertices in the full graph that are connected to a given term vertex. Further, term vertices with different output patterns shall be colored differently. Figure 2(c) illustrates the reduced graph for the two-input XOR function.

All the minterms and maxterms of the Boolean function are used in the graph because we focus on fully-specified Boolean functions. Since all the terms are used, and there are $2^{n}$ terms for an $n$-input function, the time and space complexity of our algorithm is $\Omega\left(2^{n}\right)$.

\subsection{Discussion}

Compared with other symmetry-detection methods, the symmetry-detection method proposed in our work has the following advantages: (1) it can detect all possible input and output symmetries of a function, including multi- 


\begin{tabular}{|c|c|}
\hline$x y$ & $z$ \\
\hline 00 & 0 \\
01 & 1 \\
10 & 1 \\
11 & 0 \\
\hline
\end{tabular}

(a)

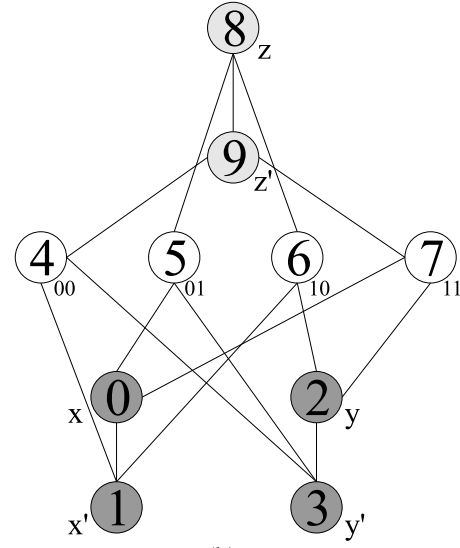

(b)

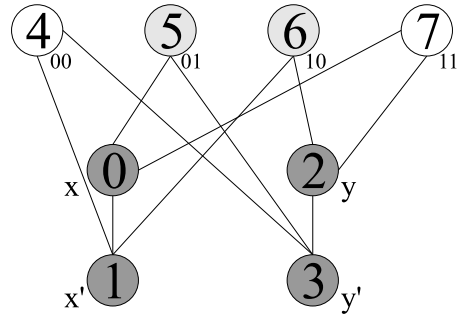

(c)

Fig. 2. Representing the 2-input XOR function by (a) the truth table, (b) the full graph, (c) the reduced graph for faster symmetry detection.

variable, higher-order and phase-shift symmetries, (2) symmetry generators are used to represent the symmetries and are very compact, and the relationship between input and output symmetries is very clear. These characteristics make the use of the symmetries easier than other methods which enumerate all symmetry pairs.

While evaluating our algorithm, we observed that Saucy is more efficient when there are few or no symmetries in the graph, in contrast, it takes more time when there are many symmetries. For example, the runtime of a randomly chosen 16-input function is $0.11 \mathrm{sec}$ because random functions typically have no symmetries. However, it takes $9.42 \mathrm{sec}$ to detect all symmetries of the 16-input XOR function. Runtimes for 18 inputs are $0.59 \mathrm{sec}$ and $92.39 \mathrm{sec}$, respectively.

\section{POST-PLACEMENT REWIRING}

This section describes two techniques for post-placement optimization - permutative rewiring and long-range rewiring. Permutative rewiring uses symmetries of extracted subcircuits to reduce wirelength. Long-range rewiring finds rewiring opportunities from equivalent wires that are farther apart. It first identifies candidate opportunities by simulating the circuit on random inputs, then prunes such opportunities based on their potential for improving interconnect and accepts only those which pass on-the-fly formal verification.

\subsection{Permutative Rewiring}

After placement, symmetries can be used to rewire the netlist to reduce the wirelength. This is achieved by reconnecting pins according to symmetries found in subcircuits, and these subcircuits are extracted as follows.

(1) We represent the netlist by a hypergraph, where cells are represented by nodes and nets are represented by hyper-edges.

(2) For each node in the hypergraph, we perform breadth-first search (BFS) starting from the node, and use the first $n$ nodes traversed as subcircuits.

(3) Similarly, we perform depth-first search (DFS) and extract subcircuits using the first $m$ nodes.

In our implementation, we perform BFS extraction 4 times with $n$ from 1 to 4 , and DFS twice with $m$ from 3 to 4. This process is capable of extracting various subcircuits suitable for rewiring. In addition to logically connected cells, min-cut placers such as Capo [Caldwell et al. 2000; Adya et al. 2004] produce a hierarchical collection of "placement bins" (buckets) that contain physically adjacent cells, and these bins are also suitable for rewiring. Currently, we also use subcircuits composed of cells in every half-bin and full-bin in our rewiring. After subcircuits are extracted, we perform symmetry detection on these subcircuits. Next, we reconnect the wires to the inputs and outputs of these subcircuits according to the detected symmetries in order to optimize wirelength.

The reason why multiple passes with different sizes of subcircuits are used is that some symmetries in small subcircuits cannot be detected in larger subcircuits. For example, in Figure 3, if the subcircuit contains all the gates, only symmetries between $x, y, z$ and $w$ can be detected, and the rewiring opportunity for $p$ and $q$ will be lost. By using multiple passes for symmetry detection, more symmetries can be extracted from the circuit.

The rewiring algorithm can be easily extended to utilize phase-shift symmetry: if the wirelength is shorter after the necessary inverters are inserted or removed, then the circuit is rewired. It can also be used to reduce the delay on critical paths. 


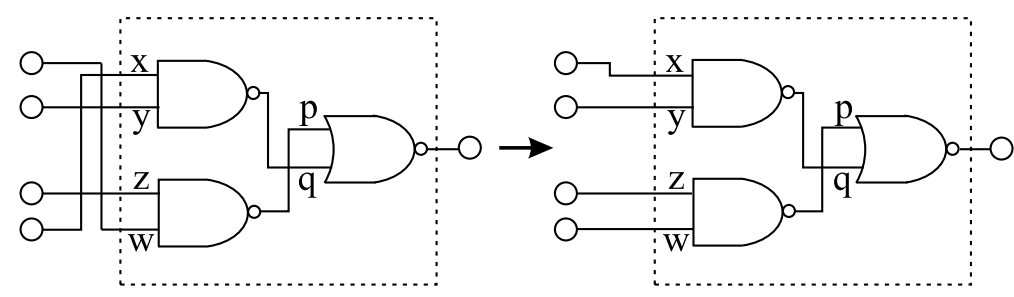

Fig. 3. A rewiring opportunity for $p$ and $q$ that cannot be detected by only considering one bucket. To rewire $p$ and $q$, a subcircuit with $p$ and $q$ as inputs must be extracted.

\subsection{Long-range Rewiring}

While permutative rewiring is effective in finding local rewiring opportunities, long-range rewiring is capable of finding equivalent wires that are farther apart. It can augment permutative rewiring to further optimize the circuit. The basic idea is similar to that popular in equivalence checking: simulation identifies potentially equivalent wires, and a CNF-SAT solver proves whether the wires are equivalent [Lu et al. 2004]. After equivalent wires are identified, we try reconnecting them and accept the reconnection that reduces wirelength. However, unlike in equivalence checking, our goal is layout optimization. Therefore we can prune rewiring opportunities before formally verifying them. In particular, we perform verification only after wirelength reduction is guaranteed, since equivalence checking is relatively slow.

\subsection{Implementation Insights}

During implementation, we observed that for subcircuits with a small number of inputs and outputs, it is more efficient to detect symmetries by enumerating all possible permutations using bit operations on the truth table. That is because the required permutations can be implemented with just a few lines of $\mathrm{C}++$ code, making this technique much faster than building the graph for Saucy. We call this algorithm naive symmetry detection. To further reduce its runtime, we limit the algorithm to detect first-order symmetries only. In our implementation, naive symmetry detection is used on subcircuits with number of inputs less than 11 and number of outputs less than 3. Experimental results show that the runtime can be reduced by more than half with almost no loss in quality, which is because the lost rewiring opportunities can be recovered in larger subcircuits where Saucy-based symmetry detection is used.

\subsection{Discussion}

Our rewiring techniques described so far use permutational symmetries. Here we describe two applications of phase-shift symmetries.

(1) The ability to handle phase-shift symmetries may reduce interconnect by enabling permutational symmetries, as the MUX example in Figure 1(c) shows.

(2) Phase-shift symmetries support metal fix of logic errors involving only inversions of signals: by reconnecting certain wires, signals may be inversed.

Compared with other rewiring techniques, the advantages of our techniques include the following:

(1) Our rewiring techniques preserve placement, therefore the effects of any change are immediately measurable. As a result, our methods are "safe" and can be applied with every flow. In other words, their application can only improve the optimization objective and never worsen it. This characteristic is especially desirable in highly-optimized circuits because changes in placements may create cell overlaps, and the legalization process to remove these overlaps may affect multiple gates, leading to a deterioration of the optimization goal.

(2) Our techniques support post-silicon metal fix, which allows reuse of transistor masks and can significantly reduce respin cost.

(3) The correctness of our optimizations can be verified easily using combinational equivalence checking.

(4) Our techniques can optimize a broad variety of objectives, as long as the objectives can be evaluated incrementally.

The limitations of our rewiring techniques include:

(1) The performance varies with each benchmark, depending on the number of symmetries and equivalent wires which exist in a design. Therefore the improvement is not guaranteed. 
(2) In permutative rewiring, the ratio of improvement tends to reduce when designs get larger. Since permutative rewiring is a local optimization, it cannot shorten global nets. However, we have not observed such a trend in long-range rewiring.

\section{EXPERIMENTAL RESULTS}

Our implementation is written in $\mathrm{C}++$, and the testcases are selected from ITC99, ISCAS and MCNC benchmarks. To better reflect modern VLSI circuits, we chose the largest testcases from each benchmark suite, and added several small and medium ones for completeness. Unselected testcases show the same trends but are omitted in this paper due to space limitation. Our experiments use the min-cut placer Capo. The platform used is Fedora 2 Linux on a Pentium-4 workstation running at $2.26 \mathrm{GHz}$ with 512M RAM.

We convert every testcase from BLIF to the Bookshelf placement format (.nodes and .nets files) using the converter provided in [Caldwell and Markov 2002; GSRCBookshelf ]. We report two different types of experimental results in this section, including the number of symmetries detected and rewiring. A flow chart of our experiments on symmetry detection and rewiring is given in Figure 4.

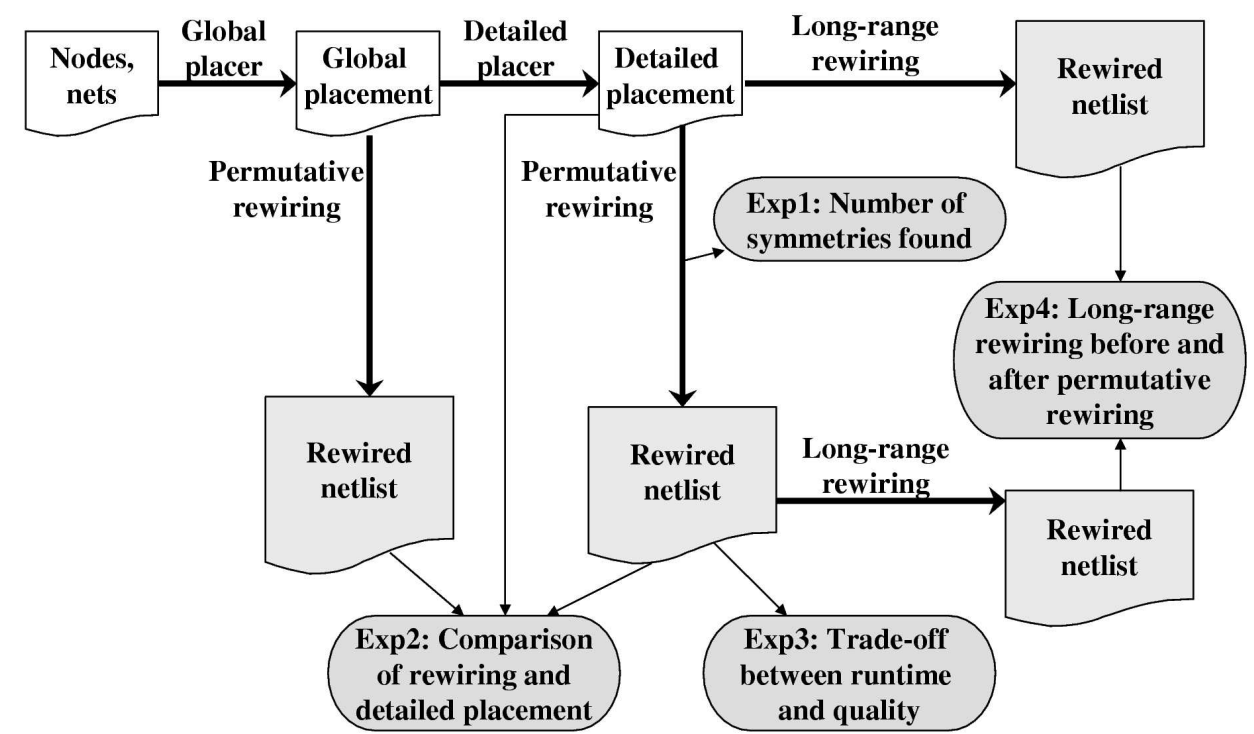

Fig. 4. Flow chart of our symmetry detection and rewiring experiments.

\subsection{Symmetries Detected}

The first experiment evaluates the symmetries found in the benchmarks, and the results are summarized in Table II. In the table, "number of subcircuits" is the number of subcircuits extracted from the benchmark for symmetry detection. "Input" is the number of subcircuits which contain input symmetries, "phase-shift input" is the number of subcircuits that contain phase-shift input symmetries. "Output" and "phase-shift output" are used in a similar way. "Input and output" are subcircuits that contain symmetries involving both inputs and outputs. The number of symmetries found in the circuits can be used to predict the probability of finding rewiring opportunities: at least $66 \%$ of the subcircuits contain permutational input symmetries and are suitable for rewiring. It can also be observed that although output symmetries do not happen as often as input symmetries, its number is not negligible and rewiring techniques should take output symmetries into consideration.

\subsection{Rewiring}

In the rewiring experiments, wirelength reduction is calculated against the original wirelength after placement using half-perimeter wirelength. The second experiment compares the wirelength reduction gained from rewiring and detailed placement. It also compares the wirelength reduction of rewiring before and after detailed placement. These results are summarized in Table III and Table IV, respectively. The maximum number of inputs allowed for symmetry detection is 16 in this experiment. From Table III, it is found that our method can effectively reduce wirelength by about $3.7 \%$, which is comparable to the improvement due to detailed-placement.

Table IV shows that the wirelength reduction is a little bit smaller when rewiring is used after detailed placement, suggesting that some rewiring opportunities interfere with optimization from detailed placement. For example, 
Table II. Number of symmetries found in benchmark circuits.

\begin{tabular}{|c|c|c|c|c|c|c|}
\hline Benchmark & \multirow{2}{*}{$\begin{array}{c}\text { Number } \\
\text { of } \\
\text { subcircuits }\end{array}$} & Input & $\begin{array}{c}\text { Phase- } \\
\text { shift } \\
\text { input }\end{array}$ & Output & $\begin{array}{c}\text { Phase- } \\
\text { shift } \\
\text { output }\end{array}$ & $\begin{array}{c}\text { Input } \\
\text { and } \\
\text { output }\end{array}$ \\
\cline { 3 - 7 } & 876 & 855 & 120 & 249 & 126 & 211 \\
\hline alu2 & 15933 & 15924 & 242 & 1245 & 243 & 1244 \\
\hline alu4 & 143 & 130 & 18 & 22 & 15 & 21 \\
\hline b02 & 1117 & 1015 & 160 & 201 & 137 & 170 \\
\hline b10 & 198544 & 190814 & 23789 & 32388 & 17559 & 24474 \\
\hline b17 & 20498 & 19331 & 9114 & 5196 & 4490 & 4145 \\
\hline c5315 & 28866 & 26626 & 12243 & 7540 & 6477 & 5895 \\
\hline c7552 & 16665 & 15506 & 6632 & 3272 & 2550 & 2852 \\
\hline dalu & 14670 & 14165 & 4298 & 3710 & 2929 & 2516 \\
\hline i10 & 141241 & 126508 & 75642 & 64973 & 59319 & 61504 \\
\hline s38417 & 122110 & 117084 & 55966 & 35632 & 29661 & 33655 \\
\hline s38584 & $100 \%$ & $94 \%$ & $28 \%$ & $23 \%$ & $18 \%$ & $20 \%$ \\
\hline \hline Average & & & & & & \\
\hline
\end{tabular}

Table III. Performance and runtime comparisons between rewiring and detailed placement.

\begin{tabular}{|c|c|c|c|c|c|c|}
\hline \multirow{2}{*}{ Benchmark } & Wirelength & \multicolumn{2}{|c|}{ Wirelength reduction } & \multicolumn{3}{|c|}{ Runtime (seconds) } \\
\cline { 3 - 7 } & & Rewiring & $\begin{array}{c}\text { Detailed } \\
\text { placement }\end{array}$ & Rewiring & $\begin{array}{c}\text { Detailed } \\
\text { placement }\end{array}$ & $\begin{array}{c}\text { Global } \\
\text { placement }\end{array}$ \\
\hline alu2.blif & 5403.29 & $3.21 \%$ & $8.98 \%$ & 2.6 & 0.2 & 3.6 \\
\hline alu4.blif & 35491.38 & $9.02 \%$ & $3.54 \%$ & 15.2 & 3 & 27.2 \\
\hline b02.blif & 142.9 & $8.29 \%$ & $0 \%$ & 2.8 & 0.4 & 0 \\
\hline b10.blif & 1548.28 & $5.04 \%$ & $3.89 \%$ & 7.2 & 0 & 1 \\
\hline b17.blif & 367223.2 & $2.92 \%$ & $2.28 \%$ & 350.6 & 32.6 & 206.2 \\
\hline c5315.blif & 30894.06 & $1.76 \%$ & $1.52 \%$ & 17.39 & 3 & 3.2 \\
\hline c7552.blif & 39226.3 & $1.71 \%$ & $1.57 \%$ & 23.8 & 4 & 2.8 \\
\hline dalu.blif & 20488.84 & $2.79 \%$ & $3.46 \%$ & 13.2 & 2.6 & 2.6 \\
\hline i10.blif & 50613.84 & $2.11 \%$ & $2.05 \%$ & 15.6 & 2.6 & 29 \\
\hline s38417.blif & 129313.2 & $2.01 \%$ & $2.05 \%$ & 180.8 & 22.2 & 17.2 \\
\hline s38584.blif & 174232.8 & $2.51 \%$ & $2.27 \%$ & 157.8 & 20.6 & 46 \\
\hline \hline Average & 77689 & $3.70 \%$ & $2.87 \%$ & 30.8 & 8.3 & 71.5 \\
\hline
\end{tabular}

detailed placement performs flipping of cells, which may interfere with permutative rewiring if the inputs of the cell are symmetric. However, the difference is very small, showing that wirelength reduction from rewiring is mostly independent of detailed placement.

Table IV. The impact of rewiring before and after detailed placement.
\begin{tabular}{|c|c|c|c|c|}
\hline Benchmark & \multicolumn{2}{|c|}{ Wirelength reduction } & \multicolumn{2}{|c|}{ Runtime (seconds) } \\
\cline { 2 - 5 } & $\begin{array}{c}\text { Before } \\
\text { detailed } \\
\text { placement }\end{array}$ & $\begin{array}{c}\text { After } \\
\text { detailed } \\
\text { placement }\end{array}$ & $\begin{array}{c}\text { Before } \\
\text { detailed } \\
\text { placement }\end{array}$ & $\begin{array}{c}\text { After } \\
\text { detailed } \\
\text { placement }\end{array}$ \\
\hline alu2 & $3.49 \%$ & $3.21 \%$ & 3.4 & 3.6 \\
\hline alu4 & $9.38 \%$ & $9.02 \%$ & 27.2 & 27.2 \\
\hline b02 & $8.29 \%$ & $8.29 \%$ & 0.2 & 0.2 \\
\hline b10 & $4.78 \%$ & $5.04 \%$ & 0.8 & 1.0 \\
\hline b17 & $3.0 \%$ & $2.92 \%$ & 199.6 & 206.2 \\
\hline c5315 & $1.71 \%$ & $1.76 \%$ & 3.6 & 3.2 \\
\hline c7552 & $1.82 \%$ & $1.71 \%$ & 2.6 & 2.8 \\
\hline dalu & $2.9 \%$ & $2.19 \%$ & 2.8 & 2.6 \\
\hline i10 & $2.05 \%$ & $2.11 \%$ & 29.2 & 29.0 \\
\hline s38417 & $2.04 \%$ & $2.01 \%$ & 18.0 & 17.2 \\
\hline s38584 & $2.50 \%$ & $2.51 \%$ & 46.2 & 46.0 \\
\hline \hline Average & $3.82 \%$ & $3.7 \%$ & 30.3 & 30.8 \\
\hline
\end{tabular}

The third experiment evaluates the relationship between the number of inputs allowed in symmetry detection, wirelength reduction and runtime. In order to show the true performance of Saucy-based symmetry detection, the 
use of naive symmetry detection is turned off in this experiment. Since our symmetry-detection method is most efficient with small number of inputs, this relationship represents the trade-off between performance and runtime. Empirical results shown in Table V indicate that the longer the rewiring program runs, the better the reduction will be. However, most improvement occurs with small number of inputs and can be achieved quickly.

Table V. The impact of the number of inputs allowed in symmetry detection on performance and runtime. The numbers are averages of all the benchmarks.

\begin{tabular}{|c|c|c|}
\hline $\begin{array}{c}\text { Number of } \\
\text { inputs allowed }\end{array}$ & $\begin{array}{c}\text { Runtime } \\
\text { (seconds) }\end{array}$ & $\begin{array}{c}\text { Wirelength } \\
\text { reduction }\end{array}$ \\
\hline 2 & 2.9 & $1.06 \%$ \\
\hline 4 & 4.3 & $2.58 \%$ \\
\hline 6 & 7.07 & $3.12 \%$ \\
\hline 8 & 14.98 & $3.5 \%$ \\
\hline 10 & 28.03 & $3.63 \%$ \\
\hline 12 & 41.34 & $3.72 \%$ \\
\hline 14 & 59.85 & $3.66 \%$ \\
\hline 16 & 82.3 & $3.68 \%$ \\
\hline
\end{tabular}

The fourth experiment shows the wirelength reduction gained from long-range rewiring and is summarized in Table VI. The SAT solver used is MiniSAT [Eén and Sörensson 2003]. For comparison, long-range rewiring is conducted before and after permutative rewiring. From the results, we can see that long-range rewiring tends to be more beneficial for larger circuits. This is because larger circuits contain more logic, therefore wires with equivalent functionality are more likely to exist. Furthermore, larger circuits contain more long wires, which make wirelength reduction more significant for many rewiring opportunities. From the comparison of long-range rewiring applied before and after permutative rewiring, we can see that the wirelength reduction gained from long-range rewiring applied after permutative rewiring is less, but the runtime is also shorter. This is because some rewiring opportunities have already been discovered by permutative rewiring. However, since permutative rewiring runs much faster, it saves time for long-range rewiring. As a result, it is clear that long-range rewiring should be applied after permutative rewiring for better performance. When long-range rewiring is applied after permutative rewiring, an extra $1.47 \%$ of wirelength reduction can be gained, which makes the total wirelength reduction $5.17 \%$ using our rewiring techniques. Considering the fact that no gate has been added or modified, our approach appears practical and very effective.

We also applied our rewiring techniques to the OpenCores suite [OpenCores ] in the IWLS'05 benchmarks [IWLSBenchmarks ], and we performed routing to measure the wirelength reduction for routed wires. The results show that our pre-routing optimizations transform into post-routing wirelength reduction effectively. Furthermore, we observe that via counts can also be reduced by our optimizations. These results show that our rewiring techniques are effective in reducing wirelength and number of vias, and they can both reduce manufacturing defects and improve yield. Reducing via count is especially important in deep sub-micron era because vias are a major cause of manufacturing faults. To study the effect of logic optimization on the performance of rewiring, we also conducted an experiment that performs rewiring on optimized and unoptimized netlists. In addition, we measured

Table VI. Wirelength reduction of long-range rewiring before and after permutative rewiring.

\begin{tabular}{|c|c|c|c|c|}
\hline \multirow[t]{2}{*}{ Benchmark } & \multicolumn{2}{|c|}{ Wirelength reduction } & \multicolumn{2}{|c|}{ Runtime (seconds) } \\
\hline & $\begin{array}{c}\text { Before } \\
\text { permutative } \\
\text { rewiring }\end{array}$ & $\begin{array}{c}\text { After } \\
\text { permutative } \\
\text { rewiring }\end{array}$ & $\begin{array}{c}\text { Before } \\
\text { permutative } \\
\text { rewiring }\end{array}$ & $\begin{array}{c}\text { After } \\
\text { permutative } \\
\text { rewiring }\end{array}$ \\
\hline alu2 & $1.63 \%$ & $1.51 \%$ & 0.66 & 0.33 \\
\hline alu4 & $0.0 \%$ & $0.05 \%$ & 13.66 & 12.66 \\
\hline b02 & $0.0 \%$ & $0.0 \%$ & 0.33 & 0.33 \\
\hline $\mathrm{b} 10$ & $0.01 \%$ & $0.0 \%$ & 0.33 & 0.33 \\
\hline b17 & $0.83 \%$ & $0.76 \%$ & 445.33 & 421.0 \\
\hline $\mathrm{c} 5315$ & $1.33 \%$ & $1.21 \%$ & 13.66 & 12.0 \\
\hline c7552 & $1.69 \%$ & $1.02 \%$ & 28.66 & 17.66 \\
\hline dalu & $2.1 \%$ & $2.21 \%$ & 23.33 & 21.66 \\
\hline $\mathrm{i} 10$ & $0.55 \%$ & $0.48 \%$ & 18.66 & 17.33 \\
\hline s38417 & $5.66 \%$ & $5.84 \%$ & 339.66 & 315.66 \\
\hline s38584 & $3.21 \%$ & $3.03 \%$ & 280.0 & 272.33 \\
\hline Average & $1.55 \%$ & $1.47 \%$ & 105.78 & 99.18 \\
\hline
\end{tabular}


the maximum delay of several benchmarks to study the effect of rewiring on circuit timing. Empirical results for these experiments are available in the Appendix.

\section{CONCLUSIONS}

In this paper we proposed a new symmetry-detection methodology and applied it to post-placement rewiring. We show experimentally that, compared with other symmetry-detection techniques, our method identifies more symmetries, including multi-variable permutational and phase-shift symmetries for both inputs and outputs. This is important in circuit rewiring because more detected symmetries create more rewiring opportunities. In addition, we proposed a long-range equivalence detector for further rewiring opportunities.

Our experimental results on common circuit benchmarks show that the wirelength reduction is comparable and orthogonal to the reduction provided by detailed placement - the reduction achieved by our method performed before and after detailed placement is similar. This shows that our rewiring method is very effective, and it should be performed after detailed placement for the best results. When applied alone, we observe an average of $3.7 \%$ wirelength reduction for the experimental benchmarks evalauted. When long-range rewiring is also applied, the total wirelength reduction increases to $5 \%$.

In summary, the rewiring technique we presented has the following advantages: (1) it does not alter the placement of any standard cells, therefore no cell overlaps are created and improvements from changes can be evaluated reliably; (2) it can be applied to a variety of existing design flows; (3) it can optimize a broad variety of objectives, such as delay and power, as long as they can be evaluated incrementally; and (4) it can easily adapt to other symmetry detectors, such as the one proposed by Chai and Kuehlmann [2006]. On the other hand, our technique has some limitations: (1) its performance depends on the specific design being optimized and there is no guarantee of wirelength reduction; and (2) the improvement tends to decrease with larger designs, similar to what has been observed for detailed placement.

\section{REFERENCES}

Adya, S. N., Chaturvedi, S., Roy, J. A., PaPa, D. A., and Markov, I. L. 2004. Unification of partitioning, placement and floorplanning. In ICCAD. 550-557.

Aloul, F. A., Ramani, A., Markov, I. L., And Sakallah, K. A. 2003. Solving difficult instances of boolean satisfiability in the presence of symmetry. IEEE Trans. on CAD of Integrated Circuits and Systems 22, 9, 1117-1137.

Bertacco, V. AND DAMiAni, M. 1997. The disjunctive decomposition of logic functions. In ICCAD. 78-82.

Caldwell, A. E., Kahng, A. B., And Markov, I. L. 2000. Can recursive bisection alone produce routable placements? In DAC. 477-482.

Caldwell, A. E. AND Markov, I. L. 2002. Toward cad-ip reuse: A web bookshelf of fundamental algorithms. IEEE Design \& Test of Computers 19, 3, 72-81.

Chai, D. AND Kuehlmann, A. 2005. Building a better boolean matcher and symmetry detector. In IWLS. 391-398.

Chai, D. and Kuehlmann, A. 2006. A compositional approach to symmetry detection in circuits. In IWLS. 228-234.

Chang, C.-W. J., Hsiao, M.-F., Hu, B., Wang, K., Marek-Sadowska, M., Cheng, C.-K., And Chen, S.-J. 2004. Fast postplacement optimization using functional symmetries. IEEE Trans. on CAD of Integrated Circuits and Systems 23, 1, 102-118.

Chang, C.-W. J. And MareK-SAdowska, M. 2001. Single-pass redundancy addition and removal. In ICCAD. 606-609.

ChANG, K.-H., MARKov, I. L., AND BerTaCCO, V. 2005. Post-placement rewiring and rebuffering by exhaustive search for functional symmetries. In ICCAD. 56-63.

Chang, K.-H., Markov, I. L., And Bertacco, V. 2006. Keeping Physical Synthesis Safe and Sound. University of Michigan Technical Report CSE-TR-522-06.

Chang, S.-C., Cheng, K.-T., Woo, N. S., And Marek-Sadowska, M. 1997. Postlayout logic restructuring using alternative wires. IEEE Trans. on CAD of Integrated Circuits and Systems 16, 6, 587-596.

Chang, S.-C., van GinneKen, L. P. P. P., AND MAReK-SAdowska, M. 1999. Circuit optimization by rewiring. IEEE Trans. Computers 48, 9, 962-970.

ConG, J. AND LONG, W. 2001. Theory and algorithm for spfd-based global rewiring. In IWLS. 150-155.

Darga, P. T., Liffiton, M. H., SAKallah, K. A., AND Markov, I. L. 2004. Exploiting structure in symmetry detection for cnf. In $D A C$. 530-534.

EÉN, N. AND SÖRENSSON, N. 2003. An extensible sat-solver. In SAT. 502-518.

GSRCBOOKSHELF. http://vlsicad.eecs.umich.edu/bk/placeutils/.

Hrkic, M., Lillis, J., AND Beraudo, G. 2004. An approach to placement-coupled logic replication. In DAC. 711-716.

IWLSBENCHMARKS. http://iwls.org/iwls2005/benchmarks.html.

JiAng, Y.-M., Krstic, A., Cheng, K.-T., AND MAReK-SADOWsKa, M. 1997. Post-layout logic restructuring for performance optimization. In DAC. 662-665.

KraVets, V. N. AND SAKALlah, K. A. 2000. Generalized symmetries in boolean functions. In ICCAD. 526-532.

Lu, F., Wang, L.-C., Cheng, K.-T. T., Moondanos, J., And Hanna, Z. 2004. A signal correlation guided circuit-sat solver. J. UCS 10, 12, 1629-1654.

MishCHENKo, A. 2003. Fast computation of symmetries in boolean functions. IEEE Trans. on CAD of Integrated Circuits and Systems 22, 11, $1588-1593$ 
OPENCORES. http://www.opencores.org/.

PAnda, S., Somenzi, F., AND Plessier, B. 1994. Symmetry detection and dynamic variable ordering of decision diagrams. In ICCAD. 628-631.

PomeranZ, I. AND REDDY, S. M. 1994. On determining symmetries in inputs of logic circuits. IEEE Trans. on CAD of Integrated Circuits and Systems 13, 11, 1428-1434.

SAUCY. http://vlsicad.eecs.umich.edu/bk/saucy/.

Wang, G., Kuehlmann, A., And Sangiovanni-Vincentelli, A. L. 2003. Structural detection of symmetries in boolean functions. In ICCD. 498-503.

WLLACE, D. E. 2001. Recognizing input equivalence in digital logic. In IWLS. 207-212.

Wu, Q., CHEN, C. Y. R., AND ACKEN, J. M. 1994. Efficent boolean matching algorithm for cell libraries. In ICCD. IEEE Computer Society, Washington, DC, USA, 36-39.

WU, Y.-L., LoNG, W., AND FAN, H. 2000. A fast graph-based alternative wiring scheme for boolean networks. In VLSI Design. $268-273$.

\section{Appendix}

Proof of Correctness of Symmetry Detection. We first prove the correctness of the reduced graph construction proposed in Section 3. Our proofs below are presented as a series of numbered steps.

(1) First, we need to prove that there is a one-to-one mapping between the function and its graph. This mapping can be defined following the graph construction in Section 3. The inverse mapping (from a graph to a function) is also given in Section 3.

(2) Second, we need to prove that there is a one-to-one mapping between symmetries of the function and automorphisms of the graph.

(a) First, we want to show that a symmetry of the function is an automorphism of the graph. Symmetry of function means that permutation of the inputs will not change the output, and permutation in inputs corresponds to reevaluation of the outputs of that the term. Since the inputs are symmetric, no output will be changed by the permutation, and the color of the term vertices in the corresponding graph will remain the same. Therefore it is also an automorphism of the graph.

(b) Next we want to show that an automorphism of the graph is a symmetry of the function. Since there is an edge between the input and its complement, mapping one input vertex, say $x$, to another vertex, say $y$, will cause $x$ 's complement map to $y$ 's complement, so Boolean consistency is preserved. Since an input vertex connect to all the term vertices that contain it, swaps between two input vertices will cause all the term vertices that connect to them being swapped according to the following rule: Suppose that input vertex $x$ swaps with input vertex $y$, then all term vertices that connect to both $x$ and $y$ will also be swapped because there is an edge between the term vertex and both $x$ and $y$. Since a swap between term vertices is legal only if they have the same color, it means all automorphisms detected in the graph will not map a term vertex to another color. And since the color of the term represents an output pattern in the Boolean function, it means the outputs of the Boolean function will not be changed. Therefore an automorphism of the graph maps to an input symmetry of the Boolean function.

(3) From Step 1 and Step 2, there is a one-to-one mapping between the function and its graph, and a one-toone mapping between the symmetries of the function and the automorphisms of the graph. Therefore the symmetry-detection method for the reduced graph is correct.

Next, the correctness of the original graph is proved below. The relationship between terms and inputs are described in the previous proof. Therefore the proof here focuses on the relationship between terms and outputs. There are three possible situations: Input symmetries that do not affect the outputs, input symmetries that affect the outputs, and output symmetries that are independent of the inputs.

(1) Input symmetries that do not affect the output: the way term vertices connect to output vertices represent an output pattern. If two term vertices have exactly the same outputs, then they will connect to the same output vertices; otherwise they will connect to at least one different output vertex. Mapping a term vertex to another term vertex which has different output pattern is invalid (except for the situation described in 2) because at least one output vertex they connect to is different, therefore the connections to output vertices behave the same as coloring in the previous proof.

(2) Input symmetries that affect the output: if all terms that connect to an output pattern can be mapped to all terms connecting to another output pattern, then the output vertices corresponding to the two patterns can also be swapped because the terms that the outputs connect to will not change after the mapping. In the mean time, the input vertices that connect to the swapped minterms will also be swapped, which represent a symmetry involving both inputs and outputs. 
Table VII. Characteristics of OpenCores benchmarks.

\begin{tabular}{|c|c|c|c|}
\hline Benchmark & Gate count & Net count & Description \\
\hline step & 226 & 231 & Stepper motor controller \\
\hline sasc & 549 & 566 & Simple asynchronous serial controller \\
\hline ac97 & 11855 & 11948 & AC 97 Controller \\
\hline usb & 12808 & 12968 & USB 1.1 Functional IP \\
\hline aes & 20795 & 21055 & Advanced Encryption Standard IP core \\
\hline pci & 16816 & 16990 & PCI Bridge \\
\hline ethernet & 46771 & 46891 & Ethernet MAC core \\
\hline
\end{tabular}

Table VIII. Wirelength and via reduction after detail routing for OpenCores benchmarks. "Perm+long" is the results of long-range rewiring applied after permutative rewiring.

\begin{tabular}{|c|c|c|c|c|c|c|}
\hline \multirow{2}{*}{ Benchmark } & \multicolumn{2}{|c|}{ Original } & \multicolumn{2}{c|}{ Wirelength reduction } & \multicolumn{2}{c|}{ Via reduction } \\
\cline { 2 - 6 } & wirelength & via count & $\begin{array}{c}\text { Permutative } \\
\text { rewiring }\end{array}$ & $\begin{array}{c}\text { Perm+long } \\
\text { rewiring }\end{array}$ & $\begin{array}{c}\text { Permutative } \\
\text { rewiring }\end{array}$ & $\begin{array}{c}\text { Perm+long } \\
\text { rewiring }\end{array}$ \\
\hline step & 6582 & 1174 & $1.02 \%$ & $5.07 \%$ & $0.94 \%$ & $2.56 \%$ \\
\hline sasc & 24471 & 3648 & $0.70 \%$ & $1.54 \%$ & $0.90 \%$ & $0.66 \%$ \\
\hline ac97 & 883291 & 84414 & $0.41 \%$ & $2.05 \%$ & $1.59 \%$ & $1.70 \%$ \\
\hline usb & 1005226 & 87848 & $0.50 \%$ & $1.87 \%$ & $2.29 \%$ & $3.49 \%$ \\
\hline aes & 1390080 & 131014 & $0.89 \%$ & $3.69 \%$ & $2.08 \%$ & $3.39 \%$ \\
\hline pci & 1483021 & 122701 & $0.26 \%$ & $1.78 \%$ & $0.25 \%$ & $0.52 \%$ \\
\hline ethernet & 7926180 & 430748 & $0.37 \%$ & $4.01 \%$ & $0.57 \%$ & $1.64 \%$ \\
\hline \hline Average & 1816979 & 123078.1 & $0.59 \%$ & $2.86 \%$ & $1.23 \%$ & $1.99 \%$ \\
\hline
\end{tabular}

(3) Output symmetries that are independent of the inputs: if two sets of output vertices connect to exactly the same term vertices, then the output vertices in the two sets can be swapped, which represent output symmetries. In this case, no term swapping is involved, so the inputs are unaffected.

OpenCores Benchmark Results. We also applied our rewiring techniques to real designs from OpenCores. Some characteristics of the benchmarks are given in Table VII, and the results are summarized in Table VIII. From the results, we can see that our techniques continue to be beneficial for these designs in terms of reduction in wirelength and via counts. However, it is observed that the improvement from permutative rewiring becomes much smaller compared with the benchmarks used in the paper, while the improvement from long-range rewiring remains roughly the same. One reason is that these benchmarks are larger then previous benchmarks. As discussed in the paper, permutative rewiring is a local optimization and its improvement tends to decrease when the design gets larger. Another reason is that the pins in the cells are close to each other and the gates are small. Since permutative rewiring is more effective for larger gates when pins are farther apart, the improvement becomes less significant. The results of reduction in via counts show that permutative rewiring is more effective than long-range rewiring in reducing via counts. The reason is that permutative rewiring can reduce local congestion and increase pin access, which in turn reduce the use of vias; while long-range rewiring does not have such effects.

Effect of Logic Optimization on Rewiring. To study the effect of logic optimization on rewiring, we selected four Register-Transfer Level (RTL) benchmarks, whose characteristics are summarized in Table IX. For logic synthesis we used Cadence RTL Compiler 4.1, configuring it with low optimization effort when generating the "unoptimized" netlists, while the "optimized" netlists were generated with high effort. We performed rewiring on both types of benchmarks, and the results are summarized in Table X. From the results, we observe that the wirelength reduction obtained using long-range rewiring was smaller for optimized netlists. The reason is that logic optimization involves merging of equivalent nodes, which reduces the opportunities for long-range rewiring. However, the wirelength reduction achieved by permutative rewiring actually increased when the design is optimized, suggesting that permutative rewiring is orthogonal to logic optimizations. Since permutative rewiring requires both physical and logical information, our results indicate that it cannot be replaced by pure physical optimizations (such as detailed placement) or pure logic optimizations (such as those performed during synthesis).

Effect of Rewiring on Circuit Timing. To study the impact of rewiring on circuit timing, we instructed the detail router (Cadence NanoRoute 4.1) to report the maximum delay of the benchmarks in Table IX (optimized version) before and after rewiring, and the results are summarized in Table XI. The results show that indiscriminate longrange rewiring may increase circuit delay because it invalidates buffer insertion performed during synthesis. As a result, it must be performed carefully to avoid delay increase in critical paths. However, circuit timing has actually improved during permutative rewiring despite the fact that timing is not considered in wirelength optimization - 
Table IX. Characteristics of benchmarks for the logic optimization experiment. Optimized netlists were produced with high optimization efforts during logic synthesis, while unoptimized netlists were produced with low efforts.

\begin{tabular}{|c|c|c|c|c|c|c|c|}
\hline \multirow{2}{*}{ Benchmark } & Description & \multicolumn{3}{|c|}{ Unoptimized } & \multicolumn{3}{c|}{ Optimized } \\
\cline { 3 - 8 } & & Gate count & Wirelength & Via count & Gate count & Wirelength & Via count \\
\hline MiniRISC & A mini-RISC CPU & 4987 & 1045619 & 91338 & 4359 & 829725 & 83309 \\
\hline MD5 & An MD5 encoder & 13311 & 3073978 & 258399 & 9181 & 1789543 & 182868 \\
\hline DLX & An MIPS-Lite CPU & 14788 & 4340807 & 320418 & 11028 & 3058868 & 248093 \\
\hline Alpha & An Alpha CPU & 38831 & 15012263 & 855910 & 30212 & 9410743 & 672399 \\
\hline
\end{tabular}

Table X. Wirelength and via reduction after detail routing for unoptimized and optimized circuits. "Perm+long" is the results of long-range rewiring applied after permutative rewiring.

\begin{tabular}{|c|c|c|c|c|c|c|c|c|}
\hline \multirow[t]{3}{*}{ Benchmark } & \multicolumn{4}{|c|}{ Unoptimized } & \multicolumn{4}{|c|}{ Optimized } \\
\hline & \multicolumn{2}{|c|}{ Wirelength reduction } & \multicolumn{2}{|c|}{ Via count reduction } & \multicolumn{2}{|c|}{ Wirelength reduction } & \multicolumn{2}{|c|}{ Via count reduction } \\
\hline & $\begin{array}{c}\text { Permutative } \\
\text { rewiring }\end{array}$ & $\begin{array}{l}\text { Perm+long } \\
\text { rewiring }\end{array}$ & $\begin{array}{c}\text { Permutative } \\
\text { rewiring }\end{array}$ & $\begin{array}{l}\text { Perm+long } \\
\text { rewiring }\end{array}$ & $\begin{array}{c}\text { Permutative } \\
\text { rewiring }\end{array}$ & $\begin{array}{c}\text { Perm+long } \\
\text { rewiring }\end{array}$ & $\begin{array}{l}\text { Permutative } \\
\text { rewiring }\end{array}$ & $\begin{array}{c}\text { Perm+long } \\
\text { rewiring }\end{array}$ \\
\hline MiniRISC & $0.50 \%$ & $5.04 \%$ & $0.87 \%$ & $1.85 \%$ & $0.58 \%$ & $1.08 \%$ & $0.88 \%$ & $1.94 \%$ \\
\hline MD5 & $0.45 \%$ & $6.29 \%$ & $1.28 \%$ & $3.05 \%$ & $0.65 \%$ & $2.93 \%$ & $1.48 \%$ & $2.80 \%$ \\
\hline DLX & $0.41 \%$ & $5.67 \%$ & $1.09 \%$ & $5.51 \%$ & $0.47 \%$ & $1.78 \%$ & $0.74 \%$ & $1.67 \%$ \\
\hline Alpha & $0.24 \%$ & $8.68 \%$ & $1.02 \%$ & $6.87 \%$ & $0.36 \%$ & $1.93 \%$ & $1.34 \%$ & $2.04 \%$ \\
\hline Average & $0.40 \%$ & $6.42 \%$ & $1.07 \%$ & $4.32 \%$ & $0.52 \%$ & $1.93 \%$ & $1.11 \%$ & $1.94 \%$ \\
\hline
\end{tabular}

Table XI. Maximum circuit delay after detail routing. "Perm+long" is the results of long-range rewiring applied after permutative rewiring.

\begin{tabular}{|c|c|c|c|}
\hline \multirow{2}{*}{ Benchmark } & \multicolumn{3}{|c|}{ Maximum delay } \\
\hline & $\begin{array}{c}\text { Before } \\
\text { rewiring (ns) }\end{array}$ & $\begin{array}{c}\text { Permutative } \\
\text { rewiring }\end{array}$ & $\begin{array}{c}\text { Perm+long } \\
\text { rewiring }\end{array}$ \\
\hline MiniRISC & 7.36 & $0.43 \%$ & 3.40 \\
\hline MD5 & 20.98 & $-0.43 \%$ & $35.05 \%$ \\
\hline$\overline{\text { DLX }}$ & 9.92 & $-9.71 \%$ & $40.21 \%$ \\
\hline Alpha & 20.86 & $0.07 \%$ & $7.36 \%$ \\
\hline \multicolumn{2}{|c|}{ Average } & $-2.41 \%$ & $21.50 \%$ \\
\hline
\end{tabular}

this is not surprising because permutative rewiring reduces the length of affected nets and does not introduce new wires. In the deep sub-micron era, power consumption and reliability are often as important as circuit timing. Our results show that permutative rewiring can safely improve both objectives without deteriorating circuit timing - shorter wires reduce dynamic power consumption, while reduction in via count makes signal delays more predictable (the resistance of Tungsten vias can vary by 20-30 times). 